\title{
MULTIPLICITY OF SOLUTIONS FOR $p$-LAPLACIAN TYPE ELLIPTIC PROBLEMS WITH ELECTROMAGNETIC FIELDS AND CRITICAL NONLINEARITY
}

\author{
ZHONGYi ZHANG
}

\begin{abstract}
We consider a class of $p$-Laplacian type elliptic problems with electromagnetic fields and critical nonlinearity in bounded domains. New results about the existence and multiplicity of solutions to these problems are obtained by using the concentration-compactness principle and variational method.
\end{abstract}

\section{Introduction}

In this paper we deal with the existence and multiplicity of solutions to the following $p$-Laplacian type elliptic problems with electromagnetic fields and critical nonlinearity:

$$
\begin{cases}{\left[g\left(\int_{\Omega}\left|\nabla_{A} u\right|^{p} d x\right)\right] \Delta_{p, A} u=\lambda h\left(x,|u|^{p}\right)|u|^{p-2} u+|u|^{p^{*}-2} u,} & x \in \Omega, \\ u=0, & x \in \partial \Omega,\end{cases}
$$

where $\Delta_{p, A} u(x):=\operatorname{div}\left(|\nabla u+i A(x) u|^{p-2}(\nabla u+i A(x) u)\right.$, here $i$ is the imaginary unit, $\Omega \subset \mathbb{R}^{N}$ is an open bounded domain with smooth boundary and $\lambda$ is a positive parameter, $p^{*}=N p /(N-p)$ is the critical exponent according to

2010 Mathematics Subject Classification. 35J60, 35B33.

Key words and phrases. p-Laplacian type problem; magnetic fields; critical growth; concentration-compactness principle; variational method. 
the Sobolev embedding. Functions $h: \bar{\Omega} \times \mathbb{R} \rightarrow \mathbb{R}, g: \mathbb{R}^{+} \rightarrow \mathbb{R}^{+}$are continuous functions that satisfy the following conditions:

(G1) There exists $\alpha_{0}>0$ such that $g(t) \geq \alpha_{0}$ for all $t \geq 0$.

(G2) There exists $\sigma$ such that $1<p / \sigma<p^{*}$ and $G(t) \geq \sigma g(t) t$ for all $t \geq 0$, where $G(t)=\int_{0}^{t} g(s) d s$.

(H1) $h(x, s) \in C(\Omega \times \mathbb{R}, \mathbb{R}), h(x,-s)=-h(x, s)$ for all $s \in \mathbb{R}$.

(H2) $\lim _{|s| \rightarrow \infty} h(x, s) / s^{\left(p^{*}-p\right) / p}=0$ uniformly for $x \in \Omega$.

(H3) $\lim _{|s| \rightarrow 0^{+}} h(x, s) / s^{1 / \sigma-1}=\infty$ uniformly for $x \in \Omega$.

There is a vast literature concerning the existence and multiplicity of solutions for (1.1) with no magnetic field, namely $A(x) \equiv 0, g(t) \equiv 1$ and $p=2$, starting from the celebrated paper by Brezis and Nirenberg [2]. For example, Li and Zou [28] obtained infinitely many solutions with odd nonlinearity. Chen and $\mathrm{Li}[7]$ established the existence of infinitely many solutions by using the minimax procedure. For more related results, we refer the interested readers to [3], [5], [14], [15], [17], [22], [34] and references therein.

On the one hand, for the special case of problem (1.1) with $A(x) \equiv 0, g(t)=$ $a t+b$ and $p=2$, equation (1.1) reduces to the following Dirichlet problem of Kirchhoff type:

$$
\left\{\begin{array}{l}
-\left(a+b \int_{\Omega}|\nabla u|^{2} d x\right) \Delta u=f(x, u), \quad x \in \Omega, \\
\left.u\right|_{\partial \Omega}=0
\end{array}\right.
$$

where $\Omega \subset \mathbb{R}^{N}$, problem (1.2) is a generalization of a model introduced by Kirchhoff [23]. More precisely, Kirchhoff proposed a model given by the equation

$$
\rho \frac{\partial^{2} u}{\partial t^{2}}-\left(\frac{\rho_{0}}{h}+\frac{E}{2 L} \int_{0}^{L}\left|\frac{\partial u}{\partial x}\right|^{2} d x\right) \frac{\partial^{2} u}{\partial x^{2}}=0,
$$

where $\rho, \rho_{0}, h, E, L$ are constants, which extends the classical D'Alembert's wave equation, by considering the effects of changes in the length of strings during vibrations. Equation (1.2) is related to the stationary analogue of problem (1.3). Problem (1.2) received much attention only after Lions [26] proposed an abstract framework to the problem. Some important and interesting results can be found in, for example, [11], [10], [18], [20], [21], [24], [30], [32], [39]. We note that the results dealing with problem (1.2) with critical nonlinearity are relatively scarce. For the case $p \neq 2$, by means of a direct variational method, the authors proved the existence and multiplicity of solutions to a class of $p$-Kirchhoff-type problem with Dirichlet boundary data [12]. In [29], the author showed the existence of infinite solutions to the $p$-Kirchhoff type quasilinear elliptic equation. But they did not give any further information on the sequence of solutions. 
For $A(x) \not \equiv 0$, there are also many works dealing with the magnetic case. The first one seems to be [13] where the existence of standing waves was obtained for $\hbar>0$ fixed and for special classes of magnetic fields. If $A$ and $W$ are periodic functions, the existence of various types of solutions for fixed $\hbar>0$ was proved in [1] by applying minimax arguments. Concerning semiclassical bound states, it is proved in [25] that for $\hbar>0$ sufficiently small there exists a least energy solution which concentrates near the global minimum of $W$. A multiplicity result for solutions was obtained in [8] by using a topological argument. There it was also proved that the magnetic potential $A$ contributes only to the phase factor of solitary solutions for $\hbar>0$ sufficiently small. In [9] single-bump bound states were considered by using perturbation methods, these concentrate near a nondegenerate critical point of $W$ as $\hbar \rightarrow 0$. For the critical growth case, Wang [37] studied the electromagnetic Schrödinger equations

$$
-(\nabla+i A(x))^{2} u(x)+\lambda V(x) u(x)=K(x)|u|^{2^{*}-2} u \quad \text { for } x \in \mathbb{R}^{N} .
$$

By applying the linking theorem twice to the corresponding functional, they established the existence results. Chabrowski and Szulkin [4] considered problems (1.2) under the assumption that $V$ changes sign, by using a minmax type argument based on a topological linking, they obtained a solution in the Sobolev space which is defined in the paper. For $K(x) \equiv 1$, Han [19] studied problem (1.4) and established the existence of nontrivial solutions in the critical case by means of variational method. For more results, we refer the reader to [35]-[37] and the references therein.

Motivated by the above, we aim to show the existence of infinitely many solutions of problem (1.1) and a sequence of infinitely many arbitrarily small solutions converging to zero, by using a new version of the symmetric mountainpass lemma due to Kajikiya [31].

To the best of our knowledge, the existence and multiplicity of solutions to problem (1.1) has not been studied so far by variational methods. As we shall see, problem (1.1) can be viewed as an elliptic equation coupled with a non-local term. The competing effect of the non-local term with the critical nonlinearity and the lack of compactness of the embedding of $W_{0}^{1, p}(\Omega, \mathbb{C})$ into the space $L^{p^{*}}(\Omega, \mathbb{C})$ prevents us from using variational methods in a standard way. Some new estimates for such Kirchhoff equation involving Palais-Smale sequences, which are key points to apply this kind of theory, are needed to be re-established. We mainly follow the idea of [16], [31]. Let us point out that although the idea was used before for other problems, the adaptation of the procedure to our problem is not trivial since due to the appearance of non-local term we must consider our problem in a suitable space and so we need more delicate estimates.

Our main result of this paper is the following. 
TheOrem 1.1. Suppose that (G1)-(G2) and (H1)-(H3) hold. Then there exists $\lambda^{*}>0$ such that for any $\lambda \in\left(0, \lambda^{*}\right)$ problem (1.1) has a sequence of nontrivial solutions $\left\{u_{n}\right\}$ and $u_{n} \rightarrow 0$ as $n \rightarrow \infty$.

\section{Preliminary lemmas}

We shall denote by $W_{0}^{1, p}(\Omega, \mathbb{C})$ the closure of $C_{0}^{\infty}(\Omega, \mathbb{C})$ under the norm induced by

$$
\|u\|^{p}=\int_{\Omega}\left|\nabla_{A} u\right|^{p} d x
$$

where $\nabla_{A} u:=\nabla u+i A u$. Similar to the diamagnetic inequality [13], we have the following inequality:

$$
\left|\nabla_{A} u(x)\right| \geq|\nabla| u(x)||, \quad \text { for } u \in W_{0}^{1, p}(\Omega, \mathbb{C}) .
$$

Indeed, since $A$ is real-valued

$$
|\nabla| u|(x)|=\left|\operatorname{Re}\left(\nabla u \frac{\bar{u}}{|u|}\right)\right|=\left|\operatorname{Re}(\nabla u+i A u) \frac{\bar{u}}{|u|}\right| \leq|\nabla u+i A u|
$$

(the bar denotes complex conjugation). Thus, if $u \in W_{0}^{1, p}(\Omega, \mathbb{C}),|u|$ belongs to the usual Sobolev space $W_{0}^{1, p}(\Omega, \mathbb{R})$. Moreover, the embedding $W_{0}^{1, p}(\Omega, \mathbb{C}) \hookrightarrow$ $L^{q}(\Omega, \mathbb{C})$ is continuous for each $1 \leq q \leq p^{*}$ and it is compact for $1 \leq q<p^{*}$.

Consider the energy functional $J: W_{0}^{1, p}(\Omega, \mathbb{C}) \rightarrow \mathbb{R}$ defined by

$$
J(u)=\frac{1}{p} G\left(\|u\|^{p}\right)-\lambda \int_{\Omega} H\left(x,|u|^{p}\right) d x-\frac{1}{p^{*}} \int_{\Omega}|u|^{p^{*}} d x .
$$

It is well-known that a critical point of $J$ is a weak solution of problem (1.1) (see [38]). Denote by $J^{\prime}$ the derivative operator of $J$ in the weak sense. Then

$$
\begin{aligned}
\left\langle J^{\prime}(u), v\right\rangle= & \operatorname{Re}\left\{g\left(\|u\|^{p}\right) \int_{\Omega}\left(\left|\nabla_{A} u\right|^{p-2} \nabla_{A} u \cdot \overline{\nabla_{A} v}\right) d x\right. \\
& \left.-\int_{\Omega}|u|^{p^{*}-2} u \bar{v} d x-\lambda \int_{\Omega} h\left(x,|u|^{p}\right)|u|^{p-2} u \bar{v} d x\right\},
\end{aligned}
$$

for all $u, v \in W_{0}^{1, p}(\Omega, \mathbb{C})$.

Hereafter, we denote by $\lambda_{1}>0$ the best constant of the compact embedding $W_{0}^{1, p}(\Omega, \mathbb{C}) \hookrightarrow L^{p}(\Omega, \mathbb{C})$ which is given by

$$
\lambda_{1}=\inf _{u \in W_{0}^{1, p}(\Omega, \mathbb{C}) \backslash\{0\}} \frac{\int_{\Omega}\left|\nabla_{A} u\right|^{p} d x}{\left(\int_{\Omega}|u|^{p} d x\right)^{1 / p}} .
$$


Denote by $S$ the best Sobolev constant of the embedding $W_{0}^{1, p}(\Omega, \mathbb{R}) \hookrightarrow L^{p^{*}}(\Omega, \mathbb{R})$ which is given by

$$
S=\inf _{u \in W_{0}^{1, p}(\Omega, \mathbb{R}) \backslash\{0\}} \frac{\int_{\Omega}|\nabla u|^{p} d x}{\left(\int_{\Omega}|u|^{p^{*}} d x\right)^{p / p^{*}}} .
$$

It is well-known that $S$ is independent of $\Omega$ and it is never achieved, except when $\Omega=\mathbb{R}^{N}$.

To use variational methods, we give some results related to the Palais-Smale compactness condition. Recall that a sequence $\left(u_{n}\right)$ is called a Palais-Smale sequence of $J$ at the level $c$ if $J\left(u_{n}\right) \rightarrow c$ and $J^{\prime}\left(u_{n}\right) \rightarrow 0$.

Definition 2.1 (see [40]). Let $X$ be a reflexive Banach space and $X^{*}$ its topological dual. The mapping $A: X \rightarrow X^{*}$ is said to have type $\left(\mathrm{S}_{+}\right)$if any sequence $u_{n}$ in $X$ satisfying $u_{n} \rightarrow u_{0}$ in $X$ and

$$
\limsup _{n \rightarrow+\infty}\left\langle A\left(u_{n}\right), u_{n}-u_{0}\right\rangle \leq 0
$$

contains a convergent subsequence.

For each $u \in W_{0}^{1, p}(\Omega, \mathbb{C})$, we define $A: W_{0}^{1, p}(\Omega, \mathbb{C}) \rightarrow W_{0}^{-1, p^{\prime}}(\Omega, \mathbb{C})$ by

$$
\langle A(u), \varphi\rangle=\int_{\Omega}|\nabla u|^{p-2} \nabla u \nabla \varphi d x, \quad \text { for all } \varphi \in W_{0}^{1, p}(\Omega, \mathbb{C}) .
$$

REmark 2.2. It is easy to prove that the operator $A$ is of type $\left(\mathrm{S}_{+}\right)$.

We recall the second concentration-compactness principle of Lions [27].

Lemma 2.3 (see [27]). Let $\left\{u_{n}\right\}$ be a weakly convergent sequence to $u$ in $W_{0}^{1, p}(\Omega, \mathbb{R})$ such that $\left|u_{n}\right|^{p^{*}} \rightarrow \nu$ and $\left|\nabla u_{n}\right| \rightarrow \mu$ in the sense of measures. Then, for some at most countable index set $I$,

(a) $\nu=|u|^{p^{*}}+\sum_{j \in I} \delta_{x_{j}} \nu_{j}, \nu_{j}>0$,

(b) $\mu \geq|\nabla u|^{p}+\sum_{j \in I} \delta_{x_{j}} \mu_{j}, \mu_{j}>0$,

(c) $\mu_{j} \geq S \nu_{j}^{p / p^{*}}$,

where $S$ is the best Sobolev constant, $x_{j} \in \mathbb{R}^{N}, \delta_{x_{j}}$ are Dirac measures at $x_{j}$ and $\mu_{j}, \nu_{j}$ are constants.

Under assumptions $\left(\mathrm{H}_{1}\right)$ and $\left(\mathrm{H}_{2}\right)$, we have

$$
h\left(x,|s|^{p}\right)|s|^{p}=o\left(|s|^{p^{*}}\right), \quad H\left(x,|s|^{p}\right)=o\left(|s|^{p^{*}}\right),
$$

which means that, for all $\varepsilon>0$, there exist $a(\varepsilon), b(\varepsilon)>0$ such that

$$
\begin{aligned}
\left.\left|h\left(x,|s|^{p}\right)\right| s\right|^{p} \mid & \leq a(\varepsilon)+\varepsilon|s|^{p^{*}}, \\
\left|H\left(x,|s|^{p}\right)\right| & \leq b(\varepsilon)+\varepsilon|s|^{p^{*}} .
\end{aligned}
$$


Hence, for some $c(\varepsilon)>0$,

$$
H\left(x,|s|^{p}\right)-\frac{\sigma}{p} h\left(x,|s|^{p}\right)|s|^{p} \leq c(\varepsilon)+\varepsilon|s|^{p^{*}} .
$$

Lemma 2.4. Suppose that (G1)-(G2) and (H1)-(H3) hold. Then, for any $\lambda>0$, the functional $J$ satisfies the local $(\mathrm{PS})_{c}$ condition in

$$
c \in\left(-\infty, \frac{p^{*} \sigma-p}{2 p p^{*}}\left(\alpha_{0} S\right)^{N / p}-\lambda c\left(\frac{p^{*} \sigma-p}{2 p p^{*} \lambda}\right)|\Omega|\right)
$$

in the following sense: if

$$
J\left(u_{n}\right) \rightarrow c<\frac{p^{*} \sigma-p}{2 p p^{*}}\left(\alpha_{0} S\right)^{N / p}-\lambda c\left(\frac{p^{*} \sigma-p}{2 p p^{*} \lambda}\right)|\Omega|
$$

and $J^{\prime}\left(u_{n}\right) \rightarrow 0$ for some sequence in $W_{0}^{1, p}(\Omega, \mathbb{C})$, then $\left\{u_{n}\right\}$ contains a subsequence converging strongly in $W_{0}^{1, p}(\Omega)$.

Proof. Let $\left\{u_{n}\right\}$ be a sequence in $W_{0}^{1, p}(\Omega, \mathbb{C})$ such that

$$
\begin{aligned}
& J\left(u_{n}\right)=\frac{1}{p} G\left(\left\|u_{n}\right\|^{p}\right)-\lambda \int_{\Omega} H\left(x,\left|u_{n}\right|^{p}\right) d x-\frac{1}{p^{*}} \int_{\Omega}\left|u_{n}\right|^{p^{*}} d x=c+o(1), \\
& \left\langle J^{\prime}\left(u_{n}\right), v\right\rangle=\operatorname{Re}\left\{g\left(\left\|u_{n}\right\|^{p}\right) \int_{\Omega}\left(\left|\nabla_{A} u_{n}\right|^{p-2} \nabla_{A} u_{n} \cdot \overline{\nabla_{A} v}\right) d x\right. \\
& \left.\quad-\int_{\Omega}\left|u_{n}\right|^{p^{*}-2} u_{n} \bar{v} d x-\lambda \int_{\Omega} h\left(x,\left|u_{n}\right|^{p}\right)\left|u_{n}\right|^{p-2} u_{n} \bar{v} d x\right\}=o(1)\left\|u_{n}\right\| .
\end{aligned}
$$

From (G2) we see that

$$
G\left(\left\|u_{n}\right\|^{p}\right)-\sigma g\left(\left\|u_{n}\right\|^{p}\right)\left\|u_{n}\right\|^{p} \geq 0 \quad \text { for all } n .
$$

By (2.4) and (2.5), we have

$$
\begin{aligned}
c+o(1) & \left\|u_{n}\right\|=J\left(u_{n}\right)-\frac{\sigma}{p}\left\langle J^{\prime}\left(u_{n}\right), u_{n}\right\rangle \\
= & \frac{1}{p} G\left(\left\|u_{n}\right\|^{p}\right)-\frac{\sigma}{p} g\left(\left\|u_{n}\right\|^{p}\right)\left\|u_{n}\right\|^{p}+\left(\frac{\sigma}{p}-\frac{1}{p^{*}}\right) \int_{\Omega}\left|u_{n}\right|^{p^{*}} d x \\
& -\lambda \int_{\Omega} H\left(x,\left|u_{n}\right|^{p}\right) d x+\frac{\sigma}{p} \lambda \int_{\Omega} h\left(x,\left|u_{n}\right|^{p}\right)\left|u_{n}\right|^{p} d x \\
\geq & \frac{p^{*} \sigma-p}{p p^{*}} \int_{\Omega}\left|u_{n}\right|^{p^{*}} d x-\lambda \int_{\Omega} H\left(x,\left|u_{n}\right|^{p}\right) d x+\frac{\sigma}{p} \lambda \int_{\Omega} h\left(x,\left|u_{n}\right|^{p}\right)\left|u_{n}\right|^{p} d x,
\end{aligned}
$$

i.e.

$$
\frac{p^{*} \sigma-p}{p p^{*}} \int_{\Omega}\left|u_{n}\right|^{p^{*}} d x \leq \lambda \int_{\Omega}\left(H\left(x,\left|u_{n}\right|^{p}\right)-\frac{\sigma}{p} h\left(x,\left|u_{n}\right|^{p}\right)\left|u_{n}\right|^{p}\right) d x+c+o(1)\left\|u_{n}\right\| .
$$

Then, by inequality (2.3), we have

$$
\left(\frac{p^{*} \sigma-p}{p p^{*}}-\lambda \varepsilon\right) \int_{\Omega}\left|u_{n}\right|^{p^{*}} d x \leq \lambda c(\varepsilon)|\Omega|+c+o(1)\left\|u_{n}\right\| .
$$


Setting $\varepsilon=\left(p^{*} \sigma-p\right) /\left(2 p p^{*} \lambda\right)$, we get

$$
\int_{\Omega}\left|u_{n}\right|^{p^{*}} d x \leq M+o(1)\left\|v_{n}\right\|
$$

where $o(1) \rightarrow 0$ and $M$ is some positive number. On the other hand, by (2.2) and (2.6), we have

$$
\begin{aligned}
c+o(1)\left\|u_{n}\right\| & =J\left(u_{n}\right) \\
& =\frac{1}{p} G\left(\left\|u_{n}\right\|^{p}\right)-\lambda \int_{\Omega} H\left(x,\left|u_{n}\right|^{p}\right) d x-\frac{1}{p^{*}} \int_{\Omega}\left|u_{n}\right|^{p^{*}} d x \\
& \geq \frac{\alpha_{0} \sigma}{p}\left\|u_{n}\right\|^{p}-\lambda b(\varepsilon)|\Omega|-\left[\frac{1}{p^{*}}+\lambda \varepsilon\right] \int_{\Omega}\left|u_{n}\right|^{p^{*}} d x .
\end{aligned}
$$

Therefore, (2.6) and (2.7) imply that $\left\{u_{n}\right\}$ is bounded in $W_{0}^{1, p}(\Omega, \mathbb{C})$. Hence, by the diamagnetic inequality, $\left\{\left|u_{n}\right|\right\}$ is bounded in $W_{0}^{1, p}(\Omega, \mathbb{R})$. Then, for some subsequence, there is $u \in W_{0}^{1, p}(\Omega, \mathbb{C})$ such that $u_{n} \rightarrow u$ in $W_{0}^{1, p}(\Omega, \mathbb{C})$. We claim that

$$
\int_{\Omega}\left|u_{n}\right|^{p^{*}} d x \rightarrow \int_{\Omega}|u|^{p^{*}} d x .
$$

In order to prove this claim, we suppose that

$$
\left.\left.|\nabla| u_{n}\right|^{p} \rightarrow|\nabla| u\right|^{p}+\mu \quad \text { and } \quad\left|u_{n}\right|^{p^{*}} \rightarrow|u|^{p^{*}}+\nu \quad \text { (weak* sense of measures). }
$$

Using the concentration compactness-principle, we obtain a countable index set $I$, sequences $\left\{x_{j}\right\} \subset \mathbb{R}^{N}$, and $\left\{\mu_{j}\right\},\left\{\nu_{j}\right\} \subset(0, \infty)$ such that

$$
\nu=\sum_{j \in I} \delta_{x_{j}} \nu_{j}, \quad \mu \geq \sum_{j \in I} \delta_{x_{j}} \mu_{j} \quad \text { and } \quad \mu_{j} \geq S \nu_{j}^{p / p^{*}}
$$

for all $j \in I$, where $\delta_{x_{j}}$ is the Dirac measure mass at $x_{j} \in \bar{\Omega}$. Let $\psi \in$ $C_{0}^{\infty}\left(\mathbb{R}^{N},[0,1]\right)$ such that $0 \leq \psi \leq 1$,

$$
\psi(x)= \begin{cases}1 & \text { if }|x|<1 \\ 0 & \text { if }|x| \geq 2\end{cases}
$$

and $|\nabla \psi|_{\infty} \leq 2$

For $\varepsilon>0$ and $j \in I$, denote $\psi_{\varepsilon}^{j}(x)=\psi\left(\left(x-x_{j}\right) / \varepsilon\right)$. Since $\left\{u_{n} \psi_{\varepsilon}^{j}\right\}$ is bounded in $W_{0}^{1, p}(\Omega, \mathbb{C})$ and $\psi_{\varepsilon}^{j}$ takes values in $\mathbb{R}$, a direct calculation shows that

$$
\left\langle J^{\prime}\left(u_{n}\right), \psi_{\varepsilon}^{j} u_{n}\right\rangle \rightarrow 0 \quad \text { and } \quad \overline{\nabla_{A}\left(u_{n} \psi_{\varepsilon}^{j}\right)}=i \overline{u_{n}} \nabla \psi_{\varepsilon}^{j}+\psi_{\varepsilon}^{j} \overline{\nabla_{A} u_{n}}
$$


that is,

$$
\begin{aligned}
& \text { (2.11) } g\left(\left\|u_{n}\right\|^{p}\right) \int_{\Omega}\left|\nabla_{A} u_{n}\right|^{p} \psi_{\varepsilon}^{j} d x \\
& =-g\left(\left\|u_{n}\right\|^{p}\right) \operatorname{Re}\left(\int_{\mathbb{R}^{N}} i\left|\nabla_{A} u_{n}\right|^{p-2} \overline{u_{n}} \nabla_{A} u_{n} \overline{\nabla_{A} \psi_{\varepsilon}^{j}} d x\right) \\
& +\lambda \int_{\Omega} h\left(x,\left|u_{n}\right|^{p}\right)\left|u_{n}\right|^{p} \psi_{\varepsilon}^{j} d x+\int_{\Omega}\left|u_{n}\right|^{p^{*}} \psi_{\varepsilon}^{j} d x+o_{n}(1) .
\end{aligned}
$$

Hence, by Hölder's inequality, we obtain

$$
\begin{aligned}
\text { (2.12) }\left.\limsup _{n \rightarrow \infty}\left|\operatorname{Re} \int_{\mathbb{R}^{N}} i\right| \nabla_{A} u_{n}\right|^{p-2} \overline{u_{n}} \nabla_{A} u_{n} \overline{\nabla \psi_{\varepsilon}^{j}} d x \mid \\
\leq \limsup _{n \rightarrow \infty}\left(\int_{\mathbb{R}^{N}}\left|\nabla_{A} u_{n}\right|^{p} d x\right)^{(p-1) / p}\left(\left.\int_{\mathbb{R}^{N}} \overline{\mid u_{n} \nabla_{A} \psi_{\varepsilon}^{j}}\right|^{p} d x\right)^{1 / p} \\
\leq C_{1}\left(\int_{B\left(x_{j}, 2 \varepsilon\right)}|u|^{p}\left|\nabla_{A} \psi_{\varepsilon}^{j}\right|^{p} d x\right)^{1 / p} \\
\leq C_{1}\left(\int_{B\left(x_{j}, 2 \varepsilon\right)}\left|\nabla_{A} \psi_{\varepsilon}^{j}\right|^{N} d x\right)^{1 / N}\left(\int_{B\left(x_{j}, 2 \varepsilon\right)}|u|^{p^{*}} d x\right)^{1 / p^{*}} \\
\leq C_{2}\left(\int_{B\left(x_{j}, 2 \varepsilon\right)}|u|^{p^{*}} d x\right)^{1 / p^{*}} \rightarrow 0 \text { as } \varepsilon \rightarrow 0 .
\end{aligned}
$$

Since $\psi_{\varepsilon}^{j}$ has compact support, letting $n \rightarrow \infty$ in (2.11), we deduce from (2.11) and (2.12) that

$\alpha_{0} \int_{\Omega} \psi_{\varepsilon}^{j} d \mu \leq C_{2}\left(\int_{B\left(x_{j}, 2 \varepsilon\right)}|u|^{p^{*}} d x\right)^{1 / p^{*}}+\lambda \int_{B\left(x_{j}, 2 \varepsilon\right)} h\left(x,|u|^{p}\right)|u|^{p} d x+\int_{\Omega} \psi_{\varepsilon}^{j} d \nu$.

Letting $\varepsilon \rightarrow 0$, we obtain $\alpha_{0} \mu_{j} \leq \nu_{j}$. Therefore,

$$
\left(\alpha_{0} S\right)^{N / p} \leq \nu_{j} .
$$

We will prove that this inequality is not possible. Let us assume that $\left(\alpha_{0} S\right)^{N / p} \leq$ $\nu_{j_{0}}$ for some $j_{0} \in I$. Since

$$
c=J\left(u_{n}\right)-\frac{\sigma}{p}\left\langle J^{\prime}\left(u_{n}\right), u_{n}\right\rangle+o_{n}(1),
$$

it follows that

$$
\begin{aligned}
c & =\lim _{n \rightarrow \infty}\left(J\left(u_{n}\right)-\frac{\sigma}{p}\left\langle J^{\prime}\left(u_{n}\right), u_{n}\right\rangle\right) \\
& \geq\left(\frac{\sigma}{p}-\frac{1}{p^{*}}\right) \int_{\Omega}\left|u_{n}\right|^{p^{*}} d x-\lambda \int_{\Omega}\left[H\left(x,\left|u_{n}\right|^{p}\right)-\frac{\sigma}{p} h\left(x,\left|u_{n}\right|^{p}\right)\left|u_{n}\right|^{p}\right] d x \\
& \geq\left(\frac{p^{*} \sigma-p}{p p^{*}}-\lambda \varepsilon\right) \int_{\Omega} \psi_{\varepsilon}^{j_{0}}\left|u_{n}\right|^{p^{*}} d x-\lambda c(\varepsilon)|\Omega| .
\end{aligned}
$$


Letting $\varepsilon=\left(p^{*} \sigma-p\right) /\left(2 p p^{*} \lambda\right)$ and $n \rightarrow \infty$, we obtain

$$
\begin{aligned}
c & \geq \frac{p^{*} \sigma-p}{2 p p^{*}} \sum_{j \in J} \psi_{\varepsilon}^{j_{0}}\left(x_{j}\right) \nu_{j}-\lambda c\left(\frac{p^{*} \sigma-p}{2 p p^{*} \lambda}\right)|\Omega| \\
& \geq \frac{p^{*} \sigma-p}{2 p p^{*}}\left(\alpha_{0} S\right)^{N / p}-\lambda c\left(\frac{p^{*} \sigma-p}{2 p p^{*} \lambda}\right)|\Omega| .
\end{aligned}
$$

This is impossible. Then $I=\emptyset$, and hence $u_{n} \rightarrow u$ in $L^{p^{*}}(\Omega, \mathbb{C})$.

Then, using (2.8) and the fact that $u_{n} \rightarrow u$ in $L^{p^{*}}(\Omega, \mathbb{C})$, we have

$$
\begin{aligned}
\lim _{n \rightarrow \infty} \operatorname{Re} \int_{\Omega} h\left(x,\left|u_{n}\right|^{p}\right)\left|u_{n}\right|^{p-2} \overline{\left(u_{n}-u\right)} d x & =0 \\
\lim _{n \rightarrow \infty} \operatorname{Re} \int_{\Omega}\left|u_{n}\right|^{p^{*}-2} u_{n} \overline{\left(u_{n}-u\right)} d x & =0 .
\end{aligned}
$$

From $\left\langle J^{\prime}\left(u_{n}\right), u_{n}-u\right\rangle=o_{n}(1)$, we deduce that

$$
\begin{aligned}
& \left\langle J^{\prime}\left(u_{n}\right), u_{n}-u\right\rangle=\operatorname{Re}\left\{g\left(\left\|u_{n}\right\|^{p}\right) \int_{\Omega}\left|\nabla_{A} u_{n}\right|^{p-2} \nabla_{A} u_{n} \overline{\nabla_{A}\left(u_{n}-u\right)} d x\right. \\
& \left.-\lambda \int_{\Omega} h\left(x,\left|u_{n}\right|^{p}\right)\left|u_{n}\right|^{p-2} \overline{\left(u_{n}-u\right)} d x-\int_{\Omega}\left|u_{n}\right|^{p^{*}-2} u_{n} \overline{\left(u_{n}-u\right)} d x\right\}=o_{n}(1) .
\end{aligned}
$$

This, (2.14) and (2.15) imply

$$
\lim _{n \rightarrow \infty} g\left(\left\|u_{n}\right\|^{p}\right) \operatorname{Re} \int_{\Omega}\left|\nabla_{A} u_{n}\right|^{p-2} \nabla u_{n} \overline{\nabla_{A}\left(u_{n}-u\right)} d x=0 .
$$

Since $u_{n}$ is bounded and $g$ is continuous, up to subsequence, there is $t_{0} \geq 0$ such that

$$
g\left(\left\|u_{n}\right\|^{p}\right) \rightarrow g\left(t_{0}^{p}\right) \geq \alpha_{0}, \quad \text { as } n \rightarrow \infty
$$

and so

$$
\lim _{n \rightarrow \infty} \operatorname{Re} \int_{\Omega}\left|\nabla_{A} u_{n}\right|^{p-2} \nabla_{A} u_{n} \overline{\nabla_{A}\left(u_{n}-u\right)} d x=0 .
$$

Thus, by the $\left(\mathrm{S}_{+}\right)$property, $u_{n} \rightarrow u$ strongly in $W_{0}^{1, p}(\Omega, \mathbb{C})$.

\section{Existence of a sequence of arbitrarily small solutions}

In this section, we prove the existence of infinitely many solutions of (1.1) which tend to zero. Let $X$ be a Banach space and denote

$\Sigma=\{A \subset X \backslash\{0\}: A$ is closed in $X$ and symmetric with respect to the orgin $\}$.

For $A \in \Sigma$, we define genus $\gamma(A)$ as

$$
\gamma(A)=\inf \left\{m \in N: \exists \varphi \in C\left(A, \mathbb{R}^{m} \backslash\{0\},-\varphi(x)=\varphi(-x)\right)\right\} .
$$

If there is no mapping $\varphi$ as above for any $m \in N$, then $\gamma(A)=+\infty$. Let $\Sigma_{k}$ denote the family of closed symmetric subsets $A$ of $X$ such that $0 \notin A$ and $\gamma(A) \geq k$. We list some properties of the genus (see [31], [33]). 
Proposition 3.1. Let $A$ and $B$ be closed symmetric subsets of $X$ which do not contain the origin. Then the following hold:

(a) If there exists an odd continuous mapping from $A$ to $B$, then $\gamma(A) \leq$ $\gamma(B)$.

(b) If there is an odd homeomorphism from $A$ to $B$, then $\gamma(A)=\gamma(B)$.

(c) If $\gamma(B)<\infty$, then $\gamma \overline{(A \backslash B)} \geq \gamma(A)-\gamma(B)$.

(d) The $n$-dimensional sphere $S^{n}$ has a genus of $n+1$ by the Borsuk-Ulam theorem.

(e) If $A$ is compact, then $\gamma(A)<+\infty$ and there exists $\delta>0$ such that $U_{\delta}(A) \in \Sigma$ and $\gamma\left(U_{\delta}(A)\right)=\gamma(A)$, where $U_{\delta}(A)=\{x \in X:\|x-A\| \leq \delta\}$.

The following version of the symmetric mountain-pass lemma is due to Kajikiya [31].

Lemma 3.2. Let $E$ be an infinite-dimensional space, $J \in C^{1}(E, \mathbb{R})$ and suppose the following conditions hold:

$\left(\mathrm{C}_{1}\right) J$ is even, bounded from below, $J(0)=0$ and $J$ satisfies the local PalaisSmale condition, i.e. for some $\bar{c}>0$, every sequence $\left\{u_{k}\right\}$ in $E$ such that $\lim _{k \rightarrow \infty} J\left(u_{k}\right)=c<\bar{c}$ and $\lim _{k \rightarrow \infty}\left\|J^{\prime}\left(u_{k}\right)\right\|_{E^{*}}=0$ has a convergent subsequence.

$\left(\mathrm{C}_{2}\right)$ For each $k \in N$, there exists $A_{k} \in \Sigma_{k}$ such that $\sup _{u \in A_{k}} J(u)<0$.

Then either $\left(\mathrm{R}_{1}\right)$ or $\left(\mathrm{R}_{2}\right)$ below holds.

$\left(\mathrm{R}_{1}\right)$ There exists a sequence $\left\{u_{k}\right\}$ such that $J^{\prime}\left(u_{k}\right)=0, J^{\prime}\left(u_{k}\right)<0$ and $\left\{u_{k}\right\}$ converges to zero.

$\left(\mathrm{R}_{2}\right)$ There exist two sequences $\left\{u_{k}\right\}$ and $\left\{v_{k}\right\}$ such that $J^{\prime}\left(u_{k}\right)=0, J\left(u_{k}\right)<0$, $u_{k} \neq 0, \lim _{k \rightarrow \infty} u_{k}=0, J^{\prime}\left(v_{k}\right)=0, J\left(v_{k}\right)<0, \lim _{k \rightarrow \infty} v_{k}=0$, and $\left\{v_{k}\right\}$ converges to a non-zero limit.

REMARK 3.3. From Lemma 3.2 we have a sequence $\left\{u_{k}\right\}$ of critical points such that $J\left(u_{k}\right) \leq 0, u_{k} \neq 0$ and $\lim _{k \rightarrow \infty} u_{k}=0$.

In order to get infinitely many solutions we need some lemmas. Let $\varepsilon=$ $1 / p^{*} \lambda$, from (2.2) we have

$$
\begin{aligned}
J(u) & :=\frac{1}{p} G\left(\|u\|^{p}\right)-\lambda \int_{\Omega} H\left(x,|u|^{p}\right) d x-\frac{1}{p^{*}} \int_{\Omega}|u|^{p^{*}} d x \\
& \geq \frac{\alpha_{0} \sigma}{p} \int_{\Omega}\left|\nabla_{A} u\right|^{p} d x-\left(\frac{1}{p^{*}}+\varepsilon \lambda\right) \int_{\Omega}|u|^{p^{*}} d x-\lambda b(\varepsilon)|\Omega| \\
& =\frac{\alpha_{0} \sigma}{p} \int_{\Omega}\left|\nabla_{A} u\right|^{p} d x-\frac{2}{p^{*}} \int_{\Omega}|u|^{p^{*}} d x-\lambda b\left(\frac{1}{p^{*} \lambda}\right)|\Omega| \\
& \geq L_{1}\|u\|^{p}-L_{2}\|u\|^{p^{*}}-L_{3} \lambda,
\end{aligned}
$$

where $L_{1}, L_{2}, L_{3}$ are some positive constants. 
Let $Q(t)=L_{1} t^{p}-L_{2} t^{p^{*}}-L_{3} \lambda$. Then $J(u) \geq Q(\|u\|)$. Furthermore, there exists

$$
\lambda_{*}:=\frac{p L_{1}}{N L_{3}}\left(\frac{p L_{1}}{p^{*} L_{2}}\right)^{(N-p) / p}
$$

such that for $\lambda \in\left(0, \lambda_{*}\right), Q$ attains its positive maximum, that is, there exists

$$
R_{1}=\left(\frac{p L_{1}}{p^{*} L_{2}}\right)^{(N-p) / p^{2}}
$$

such that $e_{1}=Q\left(R_{1}\right)=\max _{t \geq 0} Q(t)>0$. Therefore, for $e_{0} \in\left(0, e_{1}\right)$, we may find $R_{0}<R_{1}$ such that $Q\left(R_{0}\right)=e_{0}$. Now we define

$$
\chi(t)= \begin{cases}1, & 0 \leq t \leq R_{0}, \\ \frac{L_{1} t^{p}-\lambda L_{3}-e_{1}}{L_{2} t^{p^{*}}}, & t \geq R_{1}, \\ C^{\infty}, \quad \chi(t) \in[0,1], & R_{0} \leq t \leq R_{1} .\end{cases}
$$

Then it is easy to see $\chi \in[0,1]$ and $\chi$ is $C^{\infty}$. Since the functional $J$ is not bounded from below, we could not use the theory directly. So we follow [16] to consider a truncated functional of $J$. Let $\varphi(u)=\chi(\|u\|)$ and consider the perturbation of $J$ :

$$
T(u):=\frac{1}{p} G\left(\|u\|^{p}\right)-\frac{1}{p^{*}} \varphi(u) \int_{\Omega}|u|^{p^{*}} d x-\lambda \varphi(u) \int_{\Omega} H\left(x,|u|^{p}\right) d x .
$$

Then

$$
T(u) \geq L_{1}\|u\|^{p}-L_{2} \varphi(v)\|u\|^{p^{*}}-L_{3} \lambda=\bar{Q}(\|u\|),
$$

where $\bar{Q}(t)=L_{1} t^{p}-L_{2} \chi(t) t^{p^{*}}-L_{3} \lambda$ and

$$
\bar{Q}(t)= \begin{cases}Q(t), & 0 \leq t \leq R_{0} \\ e_{1}, & t \geq R_{1}\end{cases}
$$

From the above arguments, we have the following:

Lemma 3.4. Let $T$ be defined as in (3.1). Then

(a) $T \in C^{1}\left(W_{0}^{1, p}(\Omega, \mathbb{C}), \mathbb{R}\right)$ and $T$ is even and bounded from below.

(b) If $T(u)<e_{0}$, then $\bar{Q}(\|u\|)<e_{0}$, consequently, $\|u\|<R_{0}$ and $J(u)=$ $T(u)$.

(c) There exists $\lambda^{*}$ such that, for $\lambda \in\left(0, \lambda^{*}\right)$, T satisfies a local $(\mathrm{PS})_{c}$ condition for

$$
c<e_{0} \in\left(0, \min \left\{e_{1}, \frac{p^{*} \sigma-p}{2 p p^{*}}\left(\alpha_{0} S\right)^{N / p}-\lambda c\left(\frac{p^{*} \sigma-p}{2 p p^{*} \lambda}\right)|\Omega|\right\}\right) .
$$

Lemma 3.5. Suppose that (G1)-(G2) and (H3) hold. Then for any $k \in \mathbb{N}$, there exists $\delta=\delta(k)>0$ such that $\gamma\left(\left\{u \in W_{0}^{1, p}(\Omega, \mathbb{C}): T(u) \leq-\delta(k)\right\} \backslash\{0\}\right) \geq k$. 
Proof. Firstly, by $\left(\mathrm{H}_{3}\right)$ of Theorem 1.1 , for any fixed $u \in W_{0}^{1, p}(\Omega, \mathbb{C}), u \neq 0$, we have

$$
H\left(x,|\rho u|^{p}\right) \geq M(\rho)|\rho u|^{p / \sigma} \text { with } M(\rho) \rightarrow \infty \text { as } \rho \rightarrow 0 .
$$

On the other hand, by integrating (G2), we obtain

$$
G(t) \leq \frac{G\left(t_{0}\right)}{t_{0}^{1 / \sigma}} t^{1 / \sigma}=C_{0} t^{1 / \sigma} \quad \text { for all } t \geq t_{0}>0 .
$$

Secondly, given any $k \in N$, let $E_{k}$ be a $k$-dimensional subspace of $W_{0}^{1, p}(\Omega, \mathbb{C})$. There then exists a positive constant $\delta$ such that

$$
\|u\| \leq \delta|u|_{p / \sigma} \text { for all } u \in E_{k} .
$$

Therefore for any $u \in E_{k}$ with $\|u\|=1$ and $\rho$ small enough, by (3.2) and (H3), we have

$$
\begin{aligned}
T(\rho u) & =\frac{1}{p} G\left(\|\rho u\|^{p}\right)-\frac{1}{p^{*}} \varphi(u) \int_{\Omega}|\rho u|^{p^{*}} d x-\lambda \varphi(u) \int_{\Omega} H\left(x,|\rho u|^{p}\right) d x \\
& \leq \frac{C_{0}}{p} \rho^{p / \sigma}-\frac{\lambda M(\rho)}{\delta^{p / \sigma}} \rho^{p / \sigma} \leq\left(\frac{C_{0}}{p}-\frac{\lambda M(\rho)}{\delta^{p / \sigma}}\right) \rho^{p / \sigma}=-\delta(k)<0,
\end{aligned}
$$

since $\lim _{|\rho| \rightarrow 0} M(\rho)=+\infty$. That is,

$$
\left\{u \in E_{k}:\|u\|=\rho\right\} \subset\left\{u \in W_{0}^{1, p}(\Omega, \mathbb{C}): T(u) \leq-\delta(k)\right\} \backslash\{0\} .
$$

This completes the proof.

Proof of Theorem 1.1. Recall that

$$
\Sigma_{k}=\left\{A \in W_{0}^{1, p}(\Omega, \mathbb{C}) \backslash\{0\}: A \text { is closed and } A=-A, \gamma(A) \geq k\right\}
$$

and define $c_{k}=\inf _{A \in \Sigma_{k}} \sup _{u \in A} T(u)$. By Lemmas 3.4 (a) and 3.5, we know that $-\infty<c_{k}<0$. Therefore, assumptions $\left(\mathrm{C}_{1}\right)$ and $\left(\mathrm{C}_{2}\right)$ of Lemma 3.2 are satisfied. This means that $T$ has a sequence of solutions $\left\{u_{n}\right\}$ converging to zero. Hence, Theorem 1.1 follows by Lemma 3.4 (b).

\section{A special case of problem (1.1)}

We consider the following the special case of problem (1.1):

$$
\begin{aligned}
-\left(\alpha+\beta \int_{\Omega}|\nabla u|^{p} d x\right) \Delta_{p} u & =\lambda f(x, u)+|u|^{p^{*}-2} u & & \text { in } \Omega, \\
u & =0 & & \text { on } \partial \Omega,
\end{aligned}
$$

where $\Omega$ is a bounded smooth domain of $\mathbb{R}^{N}, 1<p<N<2 p$, $\alpha$ and $\beta$ are positive constants.

Set $g(t)=\alpha+\beta t$. Then, $g(t) \geq \alpha$ and

$$
G(t)=\int_{0}^{1} g(s) d s=\alpha t+\frac{1}{2} \beta t^{2} \geq \frac{1}{2}(\alpha+\beta t) t=\sigma g(t) t,
$$


where $\sigma=1 / 2$. Hence conditions (G1) and (G2) are satisfied.

For this case, a typical example of a function satisfying conditions (F1)-(F3) is given by

$$
f(x, t)=\sum_{i=1}^{k} a_{i}(x)|t|^{q_{i}-2} t,
$$

where $k \geq 1,1<q_{i}<p / \sigma$ and $a_{i} \in C(\bar{\Omega})$. In view of Theorem 1.1, we have the following corollary.

COROLlary 4.1. Suppose that (F1)-(F3) hold. There then exists $\lambda^{*}>0$ such that for any $\lambda \in\left(0, \lambda^{*}\right)$, problem (4.1) has a sequence of nontrivial solutions $\left\{u_{n}\right\}$ and $u_{n} \rightarrow 0$ as $n \rightarrow \infty$.

\section{REFERENCES}

[1] G. Arioli And A. Szulkin, A semilinear Schrödinger equation in the presence of a magnetic field, Arch. Ration. Mech. Anal. 170 (2003), 277-295.

[2] H. Brezis and L. Nirenberg, Positive solutions of nonlinear elliptic equations involving critical exponents, Commun. Pure Appl. Math. 34 (1983), 437-477.

[3] H. Brezis And E. Lieb, A relation between pointwise convergence of functions and convergence of functionals, Proc. Amer. Math. Soc. 88 (1983), 486-490.

[4] J. Chabrowski and A. Szulkin, On the Schrödinger equation involving a critical Sobolev exponent and magnetic field, Topol. Methods Nonlinear Anal. 25 (2005), 3-21.

[5] J. Chabrowski, On multiple solutions for the nonhomogeneous $p$-Laplacian with a critical Sobolev exponent, Differential Integral Equations 8 (1995), 705-716.

[6] _ Concentration-compactness principle at infinity and semilinear elliptic equations involving critical and subcritical Sobolev exponents, Calc. Var. 3 (1995), 493-512.

[7] J. Chen AND S. LI, On multiple solutions of a singular quasi-linear equation on unbounded domain, J. Math. Anal. Appl. 275 (2002), 733-746.

[8] S. Cingolani, Semiclassical stationary states of nonlinear Schrödinger equation with external magnetic field, J. Differential Equations 188 (2003), 52-79.

[9] S. Cingolani And S. SECCHI, Semiclassical limit for nonlinear Schrödinger equation with electromagnetic fields, J. Math. Anal. Appl. 275 (2002), 108-130.

[10] F.J.S.A. CorrêA AND G.M. Figueiredo, On a elliptic equation of p-Kirchhoff type via variational methods, Bull. Austral. Math. Soc. 74 (2006), 263-277.

[11] F.J.S.A. CorRêA AND R.G. NAScimento, On a nonlocal elliptic system of p-Kirchhofftype under Neumann boundary condition, Math. Comput. Modelling 49 (2009), 598-604.

[12] G.W. Dai and R.F. HaO, Existence of solutions for a $p(x)$-Kirchhoff-type equation, J. Math. Anal. Appl. 359 (2009), 275-284.

[13] M. Esteban And P.L. Lions, Stationary solutions of nonlinear Schrödinger equations with an external magnetic field, Partial Differential Equations and the Calculus of Variations, Essays in Honor of Ennio De Giorgi, 1989, 369-408.

[14] A. Ferrero and F. Gazzola, Existence of solutions for singular critical growth semilinear elliptic equations, J. Differential Equations 177 (2001), 494-522.

[15] J. Garcia Azorero and I. Peral, Hardy inequalities and some critical elliptic and parabolic problems, J. Differential Equations 144 (1998), 441-476.

[16] _ Multiplicity of solutions for elliptic problems with critical exponent or with a nonsymmetric term, Trans. Amer. Math. Soc. 323 (1991), 877-895. 
[17] N. Ghoussoub And C. YuAn, Multiple solutions for quasi-linear PDEs involving the critical Sobolev and Hardy exponents, Trans. Amer. Math. Soc. 352 (2000), 5703-5743.

[18] A. Hamydy, M. Massar and N. Tsouli, Existence of solutions for p-Kirchhoff type problems with critical exponent, Electron. J. Differential Equations, Vol. 2011 (2011), No. 105 , pp. 1-8.

[19] P. HAN, Solutions for singular critical growth Schrödinger equation with magnetic field, Port. Math. 63 (2006), 37-45.

[20] X. He And W. Zou, Infinitely many positive solutions for Kirchhoff-type problems, Nonlinear Anal. 70 (2009), 1407-1414.

[21] _ Multiplicity of solutions for a class of Kirchhoff type problems, Acta Math. Appl. Sin. 26 (2010), 387-394.

[22] _ Infinitely many arbitrarily small solutions for sigular elliptic problems with critical Sobolev-Hardy exponents, Proc. Edinburgh Math. Soc. 52 (2009), 97-108.

[23] G. Kirchnoff, Mechanik, Teubner, Leipzig, 1883.

[24] M.A. Krasnosel'skiř, Topological Methods in the Theory of Nonlinear Integral Equations, Pergamon, Elmsford, NY, 1964.

[25] K. KuRAta, Existence and semi-classical limit of the least energy solution to a nonlinear Schrödinger equation with electromagnetic fields, Nonlinear Anal. 41 (2000), 763-778.

[26] J.L. Lions, On some equations in boundary value problems of mathematical physics, Contemporary Developments in Continuum Mechanics and Partial Differential Equations, Proc. Internat. Sympos., Inst. Mat. Univ. Fed. Rio de Janeiro, Rio de Janeiro, 1977; NorthHolland Math. Stud., vol. 30, North-Holland, Amsterdam, 1978, pp. 284-346.

[27] , The concentration compactness principle in the calculus of variations. The locally compact case. Parta I and II, Ann. Inst. H. Poincaré Anal. Non. Linéaire 1 (1984), 109-145, 223-283.

[28] S. Li AND W. Zou, Remarks on a class of elliptic problems with critical exponents, Nonlinear Anal. 32 (1998), 769-774.

[29] D.C. LiU, On a p-Kirchhoff equation via Fountain theorem and dual fountain theorem, Nonlinear Anal. 72 (2010), 302-308.

[30] T.F. Ma And J.E. Munoz Rivera, Positive solutions for a nonlinear nonlocal elliptic transmission problem, Appl. Math. Lett. 16 (2003), 243-248.

[31] R. KajikiYa, A critical-point theorem related to the symmetric mountain-pass lemma and its applications to elliptic equations, J. Funct. Anal. 225 (2005), 352-370.

[32] K. Perera and Z. Zhang, Nontrivial solutions of Kirchhoff-type problems via the Yang index, J. Differential Equations 221 (2006), 246-255.

[33] P. H. Rabinowitz, Minimax methods in critical-point theory with applications to differential equations, CBME Regional Conference Series in Mathematics, Volume 65 (American Mathematical Society, Providence, RI, 1986).

[34] E.A. Silva ANd M.S. XAVIER, Multiplicity of solutions for quasilinear elliptic problems involving critical Sobolev exponents, Annales Inst. H. Poincaré Anal. Non Linéaire 20 (2003), 341-358.

[35] Z. TANG, On the least energy solutions of nonlinear Schrödinger equations with electromagnetic fields, Comput. Math. Appl. 54 (2007), 627-637.

[36] _ Multi-bump bound states of nonlinear Schrödinger equations with electromagnetic fields and critical frequency, J. Differential Equations 245 (2008), 2723-2748.

[37] F. WANG, On an electromagnetic Schrödinger equation with critical growth, Nonlinear Anal. 69 (2008), 4088-4098.

[38] M. Willem, Minimax Theorems, Birkhäuser Boston, Boston, MA, 1996. 
[39] X. WU, Existence of nontrivial solutions and high energy solutions for SchrödingerKirchhoff-type equations in $\mathbb{R}^{N}$, Nonlinear Anal. Real World Appl. 12 (2011) 1278-1287.

[40] E. ZeidLeR, Nonlinear Function Analysis and its Applications II B, Springer-Verlag, New York, 1990.

Manuscript received December 30, 2014 accepted March 24, 2015

ZHONGYI ZHANG

College of Mathematics

Changchun Normal University

Changchun 130032, Jilin, P.R. CHINA

and

College of Mathematics

Jilin University

Changchun 130011, Jilin, P.R. CHINA

E-mail address: zhyzhang66@163.com 